\section{Changes in Physiology and Pathophysiology of Calprotectin Excretion from Neonate to Adult}

\author{
Barbara Lisowska-Myjak ${ }^{1}$ and Joanna Żytyńska-Daniluk ${ }^{2}$ \\ ${ }^{1}$ Department of Biochemistry and Clinical Chemistry, Medical University of Warsaw. Warsaw, Poland \\ ${ }^{2}$ Clinical Department of Obstetrics, Female Diseases and Gynaecological Oncology, Central Clinical Hospital of the Ministry of Interior, Warsaw, Poland
}

"Corresponding author: Barbara Lisowska-Myjak, Department of Biochemistry and Clinical Chemistry, Medical University of Warsaw, ul. Banacha 1, 02-097 Warsaw, Poland, Tel: +48 2257209 13; E-mail: basia.myjak@interia.pl

Rec date: Apr 14, 2015; Acc date: May 27, 2015; Pub date: May 30, 2015

Copyright: (c) 2015 Lisowska-Myjak B, et al. This is an open-access article distributed under the terms of the Creative Commons Attribution License, which permits unrestricted use, distribution, and reproduction in any medium, provided the original author and source are credited.

\begin{abstract}
Background: Measurement of faecal calprotectin (FCP) concentrations is a theoretically justified laboratory test used in lieu of quantitative evaluation of neutrophils penetrating into the intestinal lumen. This parameter is organspecific and identifies intestinal inflammation.
\end{abstract}

Aim: Search for individual interpretation of the cut-off values for the FCP test, taking into consideration different age groups of patients and causes of intestinal inflammation.

Methods: Review of the literature data about the calprotectin concentrations in the meconium and faeces of neonates, infants, older children and adults.

Results: FCP levels in meconium and faeces of infants are significantly higher than the concentration in older children and adults. In adults FCP is a simple and sensitive screening test for inflammatory bowel disease, also used in monitoring of disease activity and treatment efficacy. However, the diagnostic significance of high FCP levels in healthy infants and young children remains unclear.

Conclusions: FCP levels in the first year of life are higher than in adults and probably reflect 'physiological' intestinal inflammation associated with the adaptation of the neonate's intestine to life outside the uterus. On the other hand, high CP levels in meconium may indicate the important role played by neutrophils in the fetal intestine during its development.

Keywords: Calprotectin; Faeces; Meconium; Inflammation; Faecal biomarkers

\section{Introduction}

Lack of sensitive and specific, non-invasive diagnostic tests for objective, simple and reliable confirmation of intestinal inflammation in both adult and paediatric patients is an important issue in contemporary clinical practice. Biomarkers determined in blood serum are neither sensitive nor specific to inflammatory bowel disease and their elevated levels reflect a systemic inflammatory response. Faecal biomarkers may be characterized by a superior diagnostic sensitivity as they are highly organ specific. According to the current literature data, faecal calprotectin (FCP) meets the criteria of a laboratory parameter suitable for the assessment of inflammatory bowel disease $[1,2]$.

Calprotectin (CP), first described in 1980 by Fagerhol [3], is a 36 $\mathrm{kDa}$ protein of the $\mathrm{S} 100$ family (S100A8/A9 protein complex). The key property of this protein responsible for its biological activity is the ability to bind calcium and zinc. The functions of CP are associated with the regulation of inflammation and apoptosis as well as its potent antibacterial, antifungal and antiproliferative activities [4-11].
Calprotectin is mostly found in neutrophil granulocytes (it makes up $60 \%$ of the cytosolic proteins of neutrophils) and at lower concentrations in monocytes and macrophages. It may be also produced by bone marrow cells, some epithelial cells, microvascular endothelial cells and fibroblasts [4,6-10]. This is a review of literature data concerning the diagnostic value of FCP in identifying intestinal inflammation in newborns, younger and older children and adults.

\section{The Pathomechanism of Faecal Calprotectin Elevations}

The use of FCP as a biomarker of intestinal inflammation is based on the assumption that the level of FCP is proportional to the amount of neutrophils migrating across the inflamed and hence damaged mucosa into the intestinal lumen and indicative of organic intestinal disease. In active Crohn's disease and ulcerative colitis neutrophil count may increase even 10-fold [7,9,11].

FCP is a surrogate marker and its measurements are used in lieu of quantitative assessment of neutrophils migrating into the intestinal lumen. This concept was confirmed by a strong positive correlation established between the level of FCP and the count of 111-Indium labelled neutrophils, considered a 'gold standard' of faecal biomarkers for intestinal inflammation $[4,6,12,13]$. 
Due to their ability to reduce the penetration of microorganisms across the intestinal wall neutrophils present in the intestinal lumen are an important component of the intestinal barrier and an essential defence mechanism. Recruitment of neutrophils from the vascular bed into the surrounding tissue is a well-known phenomenon and it may utilized in the assessment of both acute and chronic inflammation. 'Physiological' chronic inflammation of low activity occurs in the digestive tract and probably reflects rapid and effective defence preventing potential penetration across the intestinal barrier of antigens and/or bacteria present in the intestinal lumen. At the time of inflammatory response neutrophils may additionally involve other immune cells in 'collaborative' efforts to maintain intestinal homeostasis. In spite of these theoretically explained advantages, increased recruitment and accumulation of activated neutrophils in the intestine may be associated with acute mucosal inflammation resulting in its injury $[12,13]$.

\section{Diagnostic Value of Faecal Calprotectin Assessment in Adults}

As confirmed by published studies, measurements of FCP concentrations may be used in clinical practice as a sensitive and specific biomarker to detect and assess disease activity in patients with inflammatory bowel disease (IBD). The most important advantages of using this parameter include [2,4-6,9-11]:

- Objective diagnosis of specific inflammatory processes, allowing accurate classification of patients according to type of intestinal inflammation.

- Higher specificity for diagnosing inflammatory bowel disease compared to inflammatory markers measured in serum.

- High organ specificity of faeces as a biological material to assess intestinal function.

- Determining the degree of inflammatory activity in the intestine.

- Monitoring of disease progression and response to treatment.

- Prediction of relapses.

- Non-invasive testing.

- $\mathrm{CP}$ characteristics as a easy laboratory parameter (stability in a stool sample stored at room temperature for 7 days; concentrations measured in small stool samples with commercially available tests).

\section{Role of calprotectin in differentiating between organic and functional intestinal disease}

The clinical symptoms of inflammatory intestinal diseases, such as IBD and functional intestinal disorders, such as irritable bowel syndrome (IBS) may be virtually the same which makes difficult their differential diagnosis and choice of appropriate treatment. Particularly important is the need to identify the patients most likely to require promptly further invasive investigations. High diagnostic accuracy of FCP determinations has been proven and the parameter effectively differentiates diseases associated with intestinal inflammation. On the other hand, reports of slight increases in FCP levels in patients with IBS compared to controls suggest that mild intestinal inflammation is also present in IBS patients. To date, however, optimal cut-off values for the FCP test have not been determined, which would allow a clearcut differential diagnosis distinguishing organic from non-organic intestinal disease $[4,9,14-17]$.

\section{Faecal calprotectin is not a specific method for differentiating causes of intestinal inflammation}

FCP is not a specific marker for diagnosing various factors responsible for intestinal inflammation. Increased FCP concentrations have been demonstrated in patients with IBD (Crohn's disease, ulcerative colitis) as well as in gastrointestinal infections, malignant tumours of the stomach and large intestine, polyps, damage to the small intestine induced by nonsteroidal anti-inflammatory drugs, coeliac disease and cirrhosis $[7,10,17,18]$.

Since the pathomechanism of these numerous organic diseases is associated with variable inflammation activity and hence differences in the degree of neutrophil influx into the intestinal lumen, it has been suggested FCP levels should be considered in combination with the relevant clinical diagnosis and their value interpreted as reflecting the size of neutrophil influx into the intestine. Such approach would allow an objective assessment of even mild intestinal inflammation during monitoring of IBD patients with possible prediction of relapses and evaluation of treatment efficacy. It would also facilitate assessment of intestinal inflammation due to other causes such as systemic bacterial infection, adverse effects of medication or cirrhosis $[10,13,17]$. The prerequisite of such identification of intestinal inflammation is determining the optimal cut-off values for FCP to identify patients likely to require promptly invasive investigations for IBD or any other organic disease of the intestine.

\section{Can faecal calprotectin measurements replace invasive endoscopic examination?}

Although the advantages of measuring FCP in IBD patients are numerous, it cannot replace invasive diagnostic tests in this patient population with gold standard histological examination. However, FCP may be a useful screening tool in suspected intestinal inflammation which may eventually require further prompt assessment by other methods $[6,7,10]$.

On the other hand, considering an excellent correlation between FCP levels and endoscopic assessment of IBD activity with histological sampling, this parameter may be more commonly used in clinical practice, in many cases reducing the need for endoscopic examination. Clinicians agree that in patients with confirmed intestinal inflammation FCP is a sensitive marker for disease activity and/or response to treatment while its measurements are simple and inexpensive to perform and non-invasive. FCP used as an individual test or in a panel of other inflammatory markers may replace endoscopic assessment in the monitoring of intestinal inflammation and to predict possible relapses of the condition [17-19].

\section{Diagnostic Value Calprotectin Assessment in Meconium and Faeces of Neonates and Young Children}

The physiological significance of elevated FCP levels in both fullterm and a preterm neonate in comparison with older children and adults remains unclear [20-25]. Since CP-rich leucocytes do not accumulate in the intestinal mucosa of healthy infants in the first months and in older children, it may be reasonably assumed that elevated FCP levels are accounted for by the increased migration of neutrophils into the intestinal lumen in the early period of postnatal development. The intestinal barrier which plays a key role in preventing intestinal bacteria from penetrating outside the intestinal lumen and as a result protecting against the development of systemic 
inflammation is immature at that stage. The possible causes of the 'physiological' inflammation in the neonate's intestine may include high permeability of intestinal mucosa and the development of intestinal immune defence mechanisms in response to two kinds of 'antigens': intestinal commensal bacteria and food antigens [23,26,27].

\section{The effect of commensal bacteria on faecal calprotectin concentrations in neonates and young children}

Adaptation of the neonate to life outside the uterus is closely associated with colonization of the intestine by normal bacterial flora, which explains increased FCP levels in the first weeks and months after birth compared to older patient populations. Bacteria and their potentially chemotactic products such formyl-methionyl-leucylphenylalanine (FMLP) may stimulate transepithelial migration of granulocytes into the intestinal lumen.

In both full-term and preterm neonates, exposure of the intestine to bacteria and food allergens in the early period after birth serves as the essential source of information for the developing immune system in the gastrointestinal mucosa and may have an impact on health later in life. The composition of intestinal flora in both preterm and fullterm neonates differs from that in adults and the intestinal bacterial profile begins to resemble that of adults only towards the end of the first year of life [28-30]. It has been hypothesized that characteristic properties and products of bacteria colonizing the neonate's intestine may be responsible for the specific stimulation of transepithelial granulocyte transport and release of CP. On the other hand, neutrophils in the intestinal lumen may affect the control of intestinal flora and hence the tolerance of ingested food where the neonate's immune system is less sensitive [31-35].

The pathomechanism of interactions between commensal bacteria and their effect on human development and health remain unclear. Bacterial colonization of the neonate's gastrointestinal tract has been linked to numerous significant later consequences for its health (normal brain development) and disease (cognitive and emotional disorders and such conditions as autism, type 2 diabetes, inflammatory bowel disease or gastric cancer). Also considered has been the role of bacterial colonization of the intestine or changes in the composition of normal intestinal flora produced by diet or antibiotics on the development of allergies and autoimmune diseases [33-35].

\section{Calprotectin in Neonatal Meconium}

There are two-organ specific clinical materials that can be used for the purposes of laboratory diagnosis of intestinal function in the early postnatal period. These are meconium and first stool passed after birth. These materials in differ in their components and the time of formation during the individual's development. Meconium, a thick, odourless, blackish-green content of the intestine passed by the neonate in the first 48 hours after birth, is formed exclusively during gestation and contains substances derived from the swallowed amniotic fluid, shed intestinal mucosal cells and cell degradation products from secretions of the fetal intestine. Changes in the consistency and colour to greenish-black mark the end of passing meconium and the start of excreting faeces, the composition of which depends on physiological environmental factors involved in the process of the neonate's adaptation to its life outside the uterus, including bacteria and type of feeding [20,24]. Meconium forms a unique matrix in which endo- and exogenous components which the fetus encounters during gestation are contained. High $\mathrm{CP}$ concentrations in the first meconium of both preterm neonates and healthy full-term babies confirms the hypothesis of the physiological role of neutrophils in response to the presence of bacteria or their products already in the period of fetal intestinal development in vitro [36].

\section{Is high meconium calprotectin concentration due to bacteria in meconium?}

According to a well-established opinion, the neonate's gastrointestinal tract is considered to be sterile at birth and rapidly colonized by microorganisms derived either from the mother after the baby's passage through the birth canal or from the environment. According to a more recent hypothesis, the fetus is first exposed to the maternal gastrointestinal tract flora in utero and after birth this process is continued by the same bacteria present in the breast milk. The mechanism by which bacteria are transported from the maternal intestine to the fetus and breast milk remains unclear. It has been suggested that the transport is undertaken by maternal intestinal dendritic cells which are capable of taking up bacteria from the maternal intestine and penetrating through the intercellular spaces into the circulation and then to the fetus across the placental barrier. Bacterial colonization of the fetal intestine in utero may be regarded as priming of the immune system of the fetus to adjust to life outside the uterus [27-29].

The effect of interaction between intestinal bacteria and the developing fetus may have later consequences, including the development of gastrointestinal, allergic and metabolic diseases $[28,29]$.

\section{Fecal calprotectin in the diagnosis of necrotizing enterocolitis}

Necrotizing Enterocolitis (NEC) occurs almost exclusively in premature infants. The pathomechanism of NEC is characterized by the development of inflammation with a significant involvement of neutrophils as a result of the immature response of enterocytes to bacterial stimulation and introduction of oral feeding. So far, there has been no simple laboratory test to screen for early stages of NEC and to differentiate NEC from other conditions with similar clinical symptoms, such as neonatal sepsis or focal intestinal perforation [37-39].

There is not yet any consensus in the literature concerning FCP as an appropriate marker for early diagnosis of NEC. Some authors believe that this parameter has no role in the diagnosis of NEC, especially in its early stages, because increases in FCP do not invariably precede the clinical symptoms of NEC and in many cases appear later than the radiographic evidence. On the other hand, a significant correlation of this parameter with acute NEC is important information for neonatologists because it is useful in prospective assessment of premature babies. Increasing FCP levels in serial measurements in neonates with suspected NEC may facilitate early diagnosis and help in prediction of its acute course. Diagnostic accuracy of FCP measurements in neonates with suspected NEC may be increased by including this parameter in a panel with another biomarker or biomarkers of inflammation [28,31]. 


\section{Search for Optimal Reference Values for the Diagnosis of Intestinal Inflammation in Adults, Children and Neonates}

In adults, FCP levels considered to be diagnostic of IBD vary from $50 \mu \mathrm{g} / \mathrm{g}$ to $200 \mu \mathrm{g} / \mathrm{g}$ depending on the author. The most commonly proposed upper limit of normal concentration is $50 \mu \mathrm{g} / \mathrm{g}$ but many authors are in favour of higher upper limits $(100,150,200 \mu \mathrm{g} / \mathrm{g})$ searching for such value of this parameter that would offer a greater diagnostic accuracy $[7,10,11]$.

\section{The effect of age on variations in calprotectin concentrations in meconium and faeces in children and neonates}

In the first year of life, $\mathrm{CP}$ levels in meconium and faeces of healthy infants are significantly higher than the concentrations in older children and adults and characterized by marked interindividual variations The diagnostic value of this parameter in neonates and infants below one year is at present controversial and difficult to define. Obviously, the diagnostic classification of FCP levels in neonates, infants and young children should not be based on the adult cut-off values and other, age-specific, cut-off values must be determined. Additionally, there is no agreement on the possible effect of type of feeding (breast mild, formula) on FCP which stresses the need for an individual approach to the interpretation of the parameter $[21,25]$.

The following data have been presented in the literature concerning $\mathrm{CP}$ concentrations in meconium and faeces of neonates with different gestational age, and infants and older children in different age groups.

The first 3 days after birth (meconium): Full-term infants $[\mu \mathrm{g} / \mathrm{g}]$ : mean (SD) 145 (79) [20]; median (SD) 195 (68.18) [26], Preterm infants $[\mu \mathrm{g} / \mathrm{g}]$ : median (range) 253 (9-1867) [24].

The first $2-10$ weeks after birth: Full-term infants $[\mu \mathrm{g} / \mathrm{g}]$ : median (range) 269 (31 - 2100) [23]; median (range)167 (22 - 860) [25]; median (SD) 245 (81.1) - 255 (75.4) [26]; mean (SD) 277 (109) [27], preterm infants $[\mu \mathrm{g} / \mathrm{g}]$ : mean (SD) 122.8 (98.9) [22].

The first 6 months after birth: Full-term infants $[\mu \mathrm{g} / \mathrm{g}]:$ median (range) $79(9-405)$ [23].

The first year of life: [ $\mu \mathrm{g} / \mathrm{g}]$ : median (range) $67(38-900)$ [23].

Children aged from 1 to 13 years (mean age 5.3): [mg/kg-1]: mean (SD) 40 (28) [27].

The above findings indicate the characteristic dynamics of changes in FCP levels in infants, with gradual decreases between the ages of 3 months and 5 years and a negative correlation between FCP levels and age in children aged from 1 to 13 years [27].

Despite 'physiological' high FCP levels in infants, the uses of this parameter as a candidate noninvasive marker for inflammation of the gut in neonates, especially NEC are theoretically fully justified. The recommended cut-off values for the identification of NEC range from $200 \mu \mathrm{g} / \mathrm{g}$ to $2000 \mu \mathrm{g} / \mathrm{g}$ and FCP levels $>2000 \mu \mathrm{g} / \mathrm{g}$ are strongly suggestive of NEC and other acute inflammatory states in the intestine of neonates [31,38].

Summing up, increases in FCP are a theoretically proven index of the amount of neutrophils penetrating into the intestinal lumen and an organ-specific marker of inflammation for use in routine laboratory practice. In adults, this is a simple and sensitive screening test for inflammatory bowel diseases, also useful in monitoring disease activity and treatment efficacy. In infants below one year, high FCP levels probably reflect 'physiological' inflammatory state associated with adaptation to life outside the uterus while in neonates high meconium $\mathrm{CP}$ concentrations indicate a significant role of neutrophils in the fetal intestine during its development.

\section{References}

1. Ashorn S, Honkanen T, Kolho KL, Ashorn M, Välineva T, et al. (2009) Fecal calprotectin levels and serological responses to microbial antigens among children and adolescents with inflammatory bowel disease. Inflamm Bowel Dis 15: 199-205.

2. Vieira A, Fang CB, Rolim EG, Klug WA, Steinwurz F, et al. (2009) Inflammatory bowel disease activity assessed by fecal calprotectin and lactoferrin: correlation with laboratory parameters, clinical, endoscopic and histological indexes. BMC Research Notes 2: 221-228?

3. Fagerhol MK, Dale I, Andersson T (1980) A radioimmunoassay for a granulocyte protein as a marker in studies on the turnover of such cells. Bull Eur Physiopathol Respir 16 Suppl: 273-282.

4. Gaya DR, Mackenzie JF (2002) Faecal calprotectin: a bright future for assessing disease activity in Crohn's disease. QJM 95: 557-558.

5. Vaos G, Kostakis ID, Zavras N, Chatzemichael A (2013) The role of calprotectin in pediatric disease. Biomed Res Int 2013: 542363.

6. Lewis JD (2011) The utility of biomarkers in the diagnosis and therapy of inflammatory bowel disease. Gastroenterology 140: 1817-1826.

7. Erbayrak M, Turkay C, Eraslan E, Cetinkaya H, Kasapoglu B, et al. (2009) The role of fecal calprotectin in investigating inflammatory bowel diseases. Clinics (Sao Paulo) 64: 421-425.

8. Schröder O, Naumann M, Shastri Y, Povse N, Stein J (2007) Prospective evaluation of faecal neutrophil-derived proteins in identifying intestinal inflammation: combination of parameters does not improve diagnostic accuracy of calprotectin. Aliment Pharmacol Ther 26: 1035-1042.

9. Schoepfer AM, Trummler M, Seeholzer P, Seibold-Schmid B, Seibold F (2008) Discriminating IBD from IBS: comparison of the test performance of fecal markers, blood leukocytes, CRP, and IBD antibodies. Inflamm Bowel Dis 14: 32-39.

10. Iskandar HN, Ciorba MA (2012) Biomarkers in inflammatory bowel disease: current practices and recent advances. Transl Res 159: 313-325.

11. Sipponen T, Savilahti E, Kolho KL, Nuutinen H, Turunen U, et al. (2008) Crohn's disease activity assessed by fecal calprotectin and lactoferrin: correlation with Crohn's disease activity index and endoscopic findings. Inflamm Bowel Dis 14: 40-46.

12. Colgan SP, Ehrentraut SF, Glover LE, Kominsky DJ, Campbell EL (2013) Contributions of neutrophils to resolution of mucosal inflammation. Immunol Res 55: 75-82.

13. Fournier BM, Parkos CA (2012) The role of neutrophils during intestinal inflammation. Mucosal Immunol 5: 354-366.

14. van Rheenen PF, Van de Vijver E, Fidler V (2010) Faecal calprotectin for screening of patients with suspected inflammatory bowel disease: diagnostic meta-analysis. BMJ 341: c3369.

15. D'Haens G, Ferrante M, Vermeire S, Baert F, Noman M, et al. (2012) Fecal calprotectin is a surrogate marker for endoscopic lesions in inflammatory bowel disease. Inflamm Bowel Dis 18: 2218-2224.

16. Tibble JA, Sigthorsson G, Foster R, Forgacs I, Bjarnason I (2002) Use of surrogate markers of inflammation and Rome criteria to distinguish organic from nonorganic intestinal disease. Gastroenterology 123: 450-460.

17. Lisowska-Myjak B, Muszynski J, Zborowska H (2015) Serach for the laboratory parametr sof inflammation in the serum and faeces of patients with irritable bowel syndrome. Intern J Gastroenterol Res Pract 2015: accepted for publication.

18. Gisbert JP, McNicholl AG (2009) Questions and answers on the role of faecal calprotectin as a biological marker in inflammatory bowel disease. Dig Liver Dis 41: 56-66. 
Citation: Lisowska-Myjak B, Zytynska-Daniluk J (2015) Changes in Physiology and Pathophysiology of Calprotectin Excretion from Neonate to Adult . J Mol Biomark Diagn 6: 234. doi:10.4172/2155-9929.1000234

Page 5 of 5

19. Burri E, Beglinger C (2012) Faecal calprotectin - a useful tool in the management of inflammatory bowel disease. Swiss Med Wkly 142: w13557.

20. Laforgia N, Baldassarre ME, Pontrelli G, Indrio F, Altomare MA, et al (2003) Calprotectin levels in meconium. Acta Paediatr 92: 463-466.

21. Savino F, Castagno E, Calabrese R, Viola S, Oggero R, et al. (2010) High faecal calprotectin levels in healthy, exclusively breast-fed infants. Neonatology 97: 299-304.

22. Yang Q, Smith PB, Goldberg RN, Cotten CM (2008) Dynamic change of fecal calprotectin in very low birth weight infants during the first month of life. Neonatology 94: 267-271.

23. Rugtveit J, Fagerhol MK (2002) Age-dependent variations in fecal calprotectin concentrations in children. J Pediatr Gastroenterol Nutr 34: 323-324.

24. Josefsson S, Bunn SK, Domellöf M (2007) Fecal calprotectin in very low birth weight infants. J Pediatr Gastroenterol Nutr 44: 407-413.

25. Campeotto F, Butel MJ, Kalach N, Derrieux S, Aubert-Jacquin C, et al. (2004) High faecal calprotectin concentrations in newborn infants. Arch Dis Child Fetal Neonatal Ed 89: F353-355.

26. Baldassarre ME, Altomare MA, Fanelli M, Carbone D, Di Bitonto G, et al. (2007) Does calprotectin represent a regulatory factor in host defense or a drug target in inflammatory disease? Endocr Metab Immune Disord Drug Targets 7: 1-5.

27. Olafsdottir E, Aksnes L, Fluge G, Berstad A (2002) Faecal calprotectin levels in infants with infantile colic, healthy infants, children with inflammatory bowel disease, children with recurrent abdominal pain and healthy children. Acta Paediatr 91: 45-50.

28. Neu J (2007) Gastrointestinal development and meeting the nutritional needs of premature infants. Am J Clin Nutr 85: 629S-634S.

29. Rautava S, Luoto R, Salminen S, Isolauri E (2012) Microbial contact during pregnancy, intestinal colonization and human disease. Nat Rev Gastroenterol Hepatol 10: 565-576.
30. Rougé C, Butel MJ, Piloquet H, Ferraris L, Legrand A, et al. (2010) Fecal calprotectin excretion in preterm infants during the neonatal period. PLoS One 5: e11083.

31. Campeotto F, Baldassarre M, Butel MJ, Viallon V, Nganzali F, et al. (2009) Fecal calprotectin: cutoff values for identifying intestinal distress in preterm infants. J Pediatr Gastroenterol Nutr 48: 507-510.

32. Cilieborg MS, Boye M, Sangild PT (2012) Bacterial colonization and gut development in preterm neonates. Early Hum Dev 88 Suppl 1: S41-49.

33. Thum C, Cookson AL, Otter DE, McNabb WC, Hodgkinson AJ, et al. (2012) Can nutritional modulation of maternal intestinal microbiota influence the development of the infant gastrointestinal tract? J Nutr 142: 1921-1928.

34. Moles L, Gómez M, Heilig H, Bustos G, Fuentes S, et al. (2013) Bacterial diversity in meconium of preterm neonates and evolution of their fecal microbiota during the first month of life. PLoS One 8: e66986.

35. Rhoads JM, Fatheree NY, Norori J, Liu Y, Lucke JF, et al. (2009) Altered fecal microflora and increased fecal calprotectin in infants with colic. J Pediatr 155: 823-828.

36. Jiménez E, Marín ML, Martín R, Odriozola JM, Olivares M, et al. (2008) Is meconium from healthy newborns actually sterile? Res Microbiol 159: 187-193.

37. Nanthakumar NN, Fusunyan RD, Sanderson I, Walker WA (2000) Inflammation in the developing human intestine: A possible pathophysiologic contribution to necrotizing enterocolitis. Proc Natl Acad Sci U S A 97: 6043-6048.

38. Aydemir O, Aydemir C, Sarikabadayi YU, Emre Canpolat F, Erdeve O, et al. (2012) Fecal calprotectin levels are increased in infants with necrotizing enterocolitis. J Matern Fetal Neonatal Med 25: 2237-2241.

39. Ng PC (2013) Biomarkers of necrotising enterocolitis. Sem Fetal Neonat Med. 